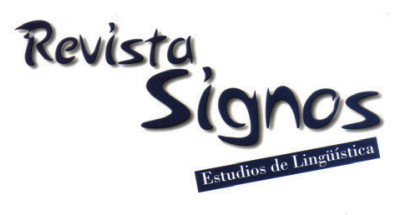

\title{
Relación entre el grado de certeza y los valores de Fo y duración silábica en algunos adverbios terminados en -mente
}

\author{
Relationship between certainty degree and F0/duration \\ syllabic values in some Spanish adverbs ending in -mente
}

\author{
Adrián Cabedo \\ Universitat de VALÈnCIA \\ Grupo Val.Es.Co \\ EsPaña \\ adrian.cabedo@uv.es
}

Recibido: 28-XII-2012 / Aceptado: 16-IX-2013

\section{Resumen}

Los nueve adverbios analizados en este artículo ('exactamente', 'indudablemente', 'efectivamente', 'evidentemente', 'obviamente', 'seguramente', 'posiblemente', 'probablemente', 'supuestamente') evalúan la certeza del enunciado al que se adjuntan; en tal sentido, el principal objetivo de este artículo es observar la relación entre el grado de certeza y el grado de marcación fónica observable en los valores de F0 y de duración silábica. Para ello, se han recogido 126 archivos de audio procedentes de corpus de español espontáneo (Corpus Val.Es.Co, COLA, Corpus de Referencia de la Lengua Española Contemporánea), se han transcrito con el programa PRAAT y, posteriormente, se han analizado estadísticamente (análisis de clúster y prueba T de Student) los registros tonales y durativos de sus respectivas sílabas. En general, los resultados obtenidos apuntan a una menor duración y a un mayor incremento tonal en adverbios que expresan cautela elocutiva y cierto valor de atenuación, como 'probablemente' o 'seguramente'.

Palabras Clave: Evidencialidad, valores epistémicos, adverbios -mente, prosodia, duración. 


\begin{abstract}
The nine adverbs examined in this study (exactamente, indudablemente, efectivamente, evidentemente, obviamente, seguramente, posiblemente, probablemente, supuestamente), evaluate the degree of certainty of a given statement. The main objective of the study was to analyze whether or not there is a relationship between the degree of certainty conveyed by each adverb and the degree of phonic markedness observed in them, expressed through factors such as pitch and syllable duration. To achieve this goal, 126 audio files collected from several spontaneous oral Spanish corpora (Val.Es.Co, COLA, CREA). Were transcribed using PRAAT software and later the tonal and durative records of each syllable were statistically analyzed (cluster analysis and Student's t-test). In general, the results show that in adverbs expressing caution and a certain mitigating value (like probablemente o seguramente), there is a) a tendency towards a shorter duration, and b) a greater tonal increase.
\end{abstract}

Key Words: Evidentiality, epistemic values, Spanish adverbs, prosody, duration.

\title{
INTRODUCCIÓN
}

En este artículo se analizan los diferentes valores de tono y de duración silábica de nueve adverbios terminados en '-mente': 'posiblemente', 'probablemente', 'supuestamente', 'indudablemente', 'seguramente', 'efectivamente', 'evidentemente', 'obviamente' y 'exactamente'. La hipótesis que se pretende contrastar es la de que un mayor o menor grado de certeza sobre el contenido del enunciado corresponde a una mayor o menor marcación prosódica.

En líneas generales, este trabajo se inserta en el marco teórico proporcionado por la escasa bibliografía que aborda esta relación pragmático-prosódica. Así, en otras lenguas, como el inglés, sí se ha atendido a la configuración prosódica de estos elementos extra-oracionales, como demuestran los trabajos conjuntos de AstrucAguilera y Nolan (2007a, 2007b). Como afirman estos autores: "the group of socalled extra-sentential elements includes phrases, (...) dislocated phrases ('They are crazy, those Romans'), and words, (...) vocatives ('Thanks, sir'), and sentential adverbs ('obviously')" (Astruc \& Nolan, 2007a: 1).

Es también presumible asumir que el comportamiento de la curva melódica pueda resultar diferencial según el distinto grado de certeza expresado por el emisor. De hecho, la intuición lingüística sugiere que la evaluación afirmativa de lo dicho, bien sea de un parlamento propio, bien de una respuesta a un turno precedente del interlocutor, pueda estar marcada prosódicamente (Briz, 1998); así, en palabras de Cornillie (2010: 302): “also, the prosody of modal expressions is to be taken more seriously if we want to fully understand the speaker's stance in conversation". Aun así, esta tarea es verdaderamente compleja, dado que, como señala Astruc (2005: 18), "sentential adverbs, as is the case with adverbs in general, constitute one of the syntactic classes which is less amenable to classification, since adverbs often have fairly idiosyncratic meanings". 
El interés fundamental de este artículo, por tanto, es analizar la relación entre la marcación prosódica y el grado de certeza o de compromiso sobre el enunciado que parecen establecer estos adverbios. De este modo, en las secciones que siguen, hemos examinado dos propiedades prosódicas de los adverbios terminados en '-mente': los valores silábicos de F0 y de duración. Estos han sido extraídos de corpus orales de español y analizados mediante pruebas de estadística multivariante, como el 'análisis de clúster' o la 'T de Student'.

\section{Marco teórico}

En español existen distintos tipos de adverbios (Kovacci, 1999; RAE, 2009; Mora, 2011): algunos poseen una forma de adjetivo ('él habla bajo'; 'él come rápido'), otros son morfológicamente adverbios ('ayer fui a tu casa'; 'fui allí para verte') y, finalmente, algunos terminan con el sufijo '-mente'. Estos últimos transmiten casi siempre valores de modo, como en 'él se fue tranquilamente' o 'él habló sinceramente'. Prosódicamente, son la única forma léxica en español que presenta dos acentos: uno en el adjetivo y otro en la sílaba '-men' (Quilis, 1993; RAE, 2010).

Los adverbios en '-mente', por lo que se refiere al nivel sintáctico y al semántico, poseen distintas extensiones o, en otras palabras, cubren, según el caso, diferentes ámbitos dentro del enunciado. En tal caso, pueden encontrarse adverbios que cubran el dictum o, por el contrario, que afecten al modus de la oración (Kovacci, 1999).

Por ejemplo, en español no es lo mismo 'ellos dijeron muchas cosas sinceramente' que 'sinceramente, ellos dijeron muchas cosas' o 'ellos dijeron muchas cosas, sinceramente'. En el primer caso, el adverbio afecta al verbo ('él habló en una manera sincera'), mientras que en los otros ejemplos la cualidad de sincero no está relacionada únicamente con el verbo, sino con el acto mismo de la enunciación ('yo digo de modo sincero que ellos dijeron muchas cosas').

Los adverbios en '-mente' pueden categorizarse pragmáticamente en 'evidenciales' o 'epistémicos' según la perspectiva teórica elegida. Cabe recordar que, en general, la 'epistemicidad' pone de manifiesto el grado de certeza que se posee sobre el contenido de un enunciado, mientras que la 'evidencialidad' señala la fuente del enunciado, bien sea el propio hablante o un emisor externo ${ }^{1}$ (Dendale \& Tasmowski, 2001; Cornillie, 2007; Pietrandea, 2007; Kotwicka, 2011; Hennemann, 2012).

Según Dendale y Tasmowski (2001), se mantiene en la actualidad una discusión teórica sobre si la 'evidencialidad' y la 'epistemicidad' son dos categorías (o modalidades) pragmáticas distintas: 'disyunción' (Willet, 1988; López, 2001); si, por el contrario, la 'evidencialidad' se incluye a modo de subcategoría en la categoría epistémica o viceversa: 'inclusión’ (Chafe \& Nichols, 1986; Nuyts, 2001; Kotwica, 2011); o si, por último, ambas modalidades, aun siendo independientes, pueden converger en algunos casos: 'solapamiento' (Plungian, 2001). 
Como muestra, la RAE (2009) establece que 'posiblemente', 'probablemente' y 'seguramente' pertenecen al grupo de los adverbios modales epistémicos, mientras que los 'obviamente', 'supuestamente', 'indudablemente', 'seguramente', 'efectivamente' y 'evidentemente' se consideran evidenciales. La 'evidencialidad' de estos últimos adverbios se manifiesta de manera dicotómica en una intensificación ('obviamente', 'indudablemente') o en una atenuación ('seguramente', 'supuestamente') de lo que se asevera.

Por ejemplo, 'supuestamente' alude a una fuente externa y atenúa asimismo el enunciado en el que se inserta (RAE, 2010), mientras que otros adverbios, como 'exactamente', 'efectivamente', 'evidentemente', etc., aluden también a una fuente externa (puede manifestarse acuerdo, aunque no necesariamente, con una elocución de otro hablante) y, al mismo tiempo, parecen intensificar el enunciado al que se adjuntan.

Por su parte, 'posiblemente' o 'probablemente' no señalan una fuente en la misma medida que los anteriores (de hecho, la RAE [2009, 2010] los considera puramente epistémicos), mientras que 'seguramente' u 'obviamente' tampoco marcan una relación tan directa con fuentes externas, dado que pueden servir como respuestas a intervenciones de otros hablantes, pero no de forma exclusiva.

Así pues, para estos adverbios es difícil calibrar la manera en la que se señala o no una fuente de conocimiento, si bien el grado de certeza sobre lo que se enuncia puede calcularse mejor. Por ello, como señala (Kotwica, 2011: 17): "la relación entre evidencialidad y modalidad epistémica en español se presenta muy ligada al contexto y, por tanto, es difícil establecerla sistemáticamente".

A efectos prácticos, se hablará de 'epistemicidad' en un sentido amplio, es decir, el componente 'evidencial' se considerará como parte de lo epistémico. En tal sentido, cabe tener en cuenta que los adverbios analizados en este trabajo manifiestan 'evidencialidad' también en mayor o menor grado, ya que, como señala Plungian, (2001: 354):

"while an evidential supplement can always be seen in an epistemic marker, the opposite does not always hold: not all evidential markers are modal in that they do not all necessarily imply an epistemic judgment".

Independientemente de la postura teórica o terminológica, los adverbios que se analizan en el presente estudio se han agrupado en una escala que indica intuitivamente una mayor o menor seguridad sobre lo afirmado: 


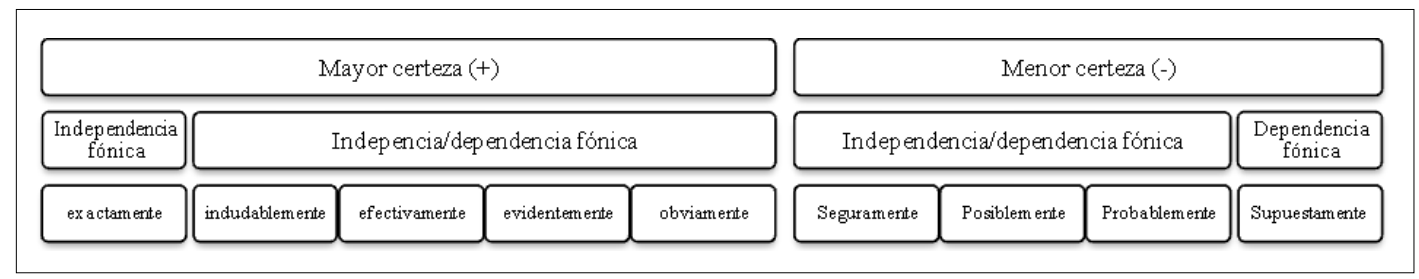

Figura 1. Relación entre la certeza y la singularidad fónica de los adverbios.

Como se observa en la Figura 1, adverbios como 'exactamente' suelen articularse fónicamente como unidades independientes y, por tanto, aparecen separados por pausas del resto de elementos ('exactamente/no quiere venir a visitarme'), mientras que otros adverbios, como supuestamente, ejemplifican lo inverso ('pues supuestamente no dijo nada de eso'). El resto de adverbios aparecen indistintamente como unidades fónicas autónomas o integradas en unidades fónicas mayores.

En español, hasta el momento, se ha prestado especial atención a la configuración morfológica o semántico-pragmática de esta clase de adverbios que terminan con el sufijo 'mente' (Azpiazu, 1999; Kovacci, 1999; Kaul de Marlangeon, 2000; Torner, 2005; Garcés, 2008; RAE, 2009, entre otros), pero se ha descuidado el apartado prosódico (Mora, 2011).

\section{Metodología}

En este apartado se expondrán las particularidades metodológicas de la investigación realizada, tanto en lo que concierne a la elección o recogida del corpus como al análisis estadístico de los datos.

Principalmente, uno de los mayores problemas que encuentra el investigador en el análisis prosódico de elementos lingüísticos es la elección de un corpus representativo. La tarea es más compleja cuando lo que se analiza es una unidad concreta, perfectamente delimitada en el contexto oral. En el caso de los nueve adverbios modales analizados en este artículo, sus límites son claros en el plano morfológico (terminación en '-mente'). No obstante, su detección resulta más difusa en el plano prosódico, más todavía si las muestras recogidas proceden de corpus orales conversacionales.

Por lo tanto, los problemas de partida de esta investigación, habituales en cualquier estudio prosódico de la conversación coloquial española (Briz, 2002; Cabedo, 2009), han sido los siguientes:

1. Análisis de material espontáneo (no grabado en laboratorio y, por tanto, sin conciencia elocutiva por parte de los interlocutores). 
2. Recopilación de un número representativo de producciones orales de adverbios en '-mente'.

3. Preservación de la calidad acústica de los archivos de audio recogidos (influidos frecuentemente por condicionamientos propios de la grabación espontánea: ruido medioambiental, lejanía de los hablantes en relación con la grabadora, distorsión acústica, etc.).

\subsection{Corpus}

En la investigación realizada se ha prestado especial atención al hecho de que el material recopilado fuese espontáneo y frecuente en español hablado (Briz, 1998). Dos de los corpus orales utilizados en esta investigación (corpus Val.Es.Co y corpus de lenguaje infantil COLA) ${ }^{2}$ están dirigidos principalmente a la obtención del español coloquial, variedad más representativa del uso lingüístico del idioma (Briz, 1998). Los adverbios encontrados en estos corpus han formado un total de 126 unidades.

Algunos adverbios ('indudablemente', 'posiblemente'), aunque con un valor semántico similar a otros ('obviamente', 'probablemente'), tienen una adscripción pragmática determinada y, por ello, son propios de registros no estrictamente coloquiales, sino más bien formales (tertulias, discurso político, etc.) (Kaul de Marlangeon, 2000; Torner, 2005). Esto conlleva que no aparezcan en los corpus mencionados anteriormente (Val.Es.Co y COLA). En consecuencia, para poder recoger datos de análisis, se ha acudido también al Corpus de Referencia de la Lengua Española Contemporánea (1992), que incluye un mayor número de material procedente de entrevistas, conversaciones, tertulias, etc.

Los resultados encontrados, según el corpus de procedencia y su frecuencia de uso, han sido los siguientes:

Tabla 1. Número de casos recogidos por corpus.

\begin{tabular}{|l|c|c|c|c|}
\hline Adverbio & Corpus COLA & Corpus Español & Corpus Val.Es.Co & Total \\
\hline efectivamente & 3 & 5 & 3 & 11 \\
\hline evidentemente & & 12 & 3 & 15 \\
\hline exactamente & 3 & & 7 & 10 \\
\hline indudablemente & & 10 & & 10 \\
\hline obviamente & 3 & 10 & & 13 \\
\hline posiblemente & & 8 & 6 & 14 \\
\hline probablemente & 4 & 5 & 5 & 14 \\
\hline seguramente & 9 & 9 & 7 & 25 \\
\hline supuestamente & 6 & 8 & & 14 \\
\hline
\end{tabular}




\subsection{Recogida de material y análisis acústico}

Dada la complejidad de la detección de elementos concretos en corpus orales, la selección de adverbios en conversaciones amplias debe combinarse necesariamente con la segmentación y la alineación silábica.

Con los archivos ya seleccionados, se han establecido tres tiers en los textgrids ${ }^{3}$ del programa PRAAT (Gráfico 1): el primero, para recoger, si es el caso, la unidad prosódico-discursiva en la que se incluye el adverbio; el segundo, por su parte, delimita el adverbio completo. Si el adverbio es una unidad prosódica completa (delimitada por pausas o por una inflexión melódica marcada) hay consonancia entre el primer y el segundo tier. Finalmente, el tercer tier divide el adverbio en sílabas.

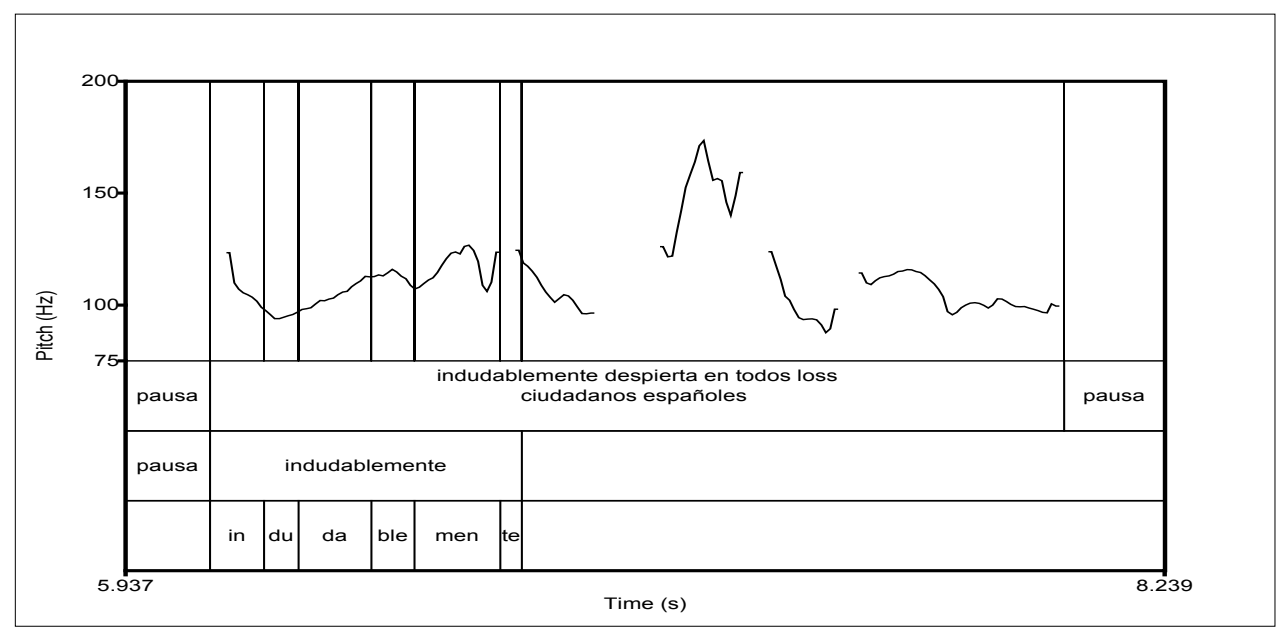

Gráfico 1. Alineación de texto y curva melódica.

Con la intención de cuantificar la curva melódica de los diferentes adverbios en '-mente', se han recogido los valores acústicos y de duración de todas las sílabas del adverbio. Con más de 100 archivos de audio en el corpus examinado y una media aproximada de 5 sílabas por adverbio (desde el más corto ['obviamente'] a los más largos ['evidentemente', 'indudablemente']), analizar los valores de F0 de cada sílaba y su duración adjunta ha supuesto realizar el estudio de 500 unidades silábicas.

En aras de agilizar el proceso de análisis, se ha utilizado el script Analyse tier ${ }^{4}$ de Daniel Hirst, que recoge los valores de F0 (media, máxima y mínima), intensidad (media, máxima y mínima) y duración para cada tier de los archivos textgrid del PRAAT y, concretamente, para cada unidad delimitada por fronteras en aquellos. De este modo, el investigador recoge resultados como los siguientes, que pueden pasar a un entorno estadístico como SPSS o R: 
Tabla 2. Valores prosódicos recogidos en la base de datos.

\begin{tabular}{|c|c|c|c|c|c|c|c|c|c|}
\hline \multicolumn{10}{|c|}{ \#Archivo creado por analyse_tier.praat versión [2009:08:07] } \\
\hline \multicolumn{10}{|c|}{ \#Autor: Daniel Hirst < daniel.hirst@lpl-aix.fr> } \\
\hline \multicolumn{10}{|c|}{$\begin{array}{l}\text { \#Análisis realizado por [Adrián Cabedo Nebot] en Martes Agosto } 16 \text { 11:58:22 } 2011 \text { en el } \\
\text { tier [sílabas] }\end{array}$} \\
\hline \multicolumn{10}{|c|}{ \#Parámetros: } \\
\hline \multicolumn{10}{|c|}{ \# Nombre de archivo : indudablemente_Acon034a } \\
\hline \multicolumn{2}{|c|}{ Archivo } & label & Duración & $\begin{array}{l}\text { F0 } \\
\text { min }\end{array}$ & $\begin{array}{l}\text { F0 } \\
\text { med }\end{array}$ & $\begin{array}{l}\text { F0 } \\
\max \end{array}$ & $\begin{array}{l}\text { Int } \\
\text { min }\end{array}$ & $\begin{array}{l}\text { Int } \\
\text { med }\end{array}$ & $\begin{array}{l}\text { Int } \\
\max \end{array}$ \\
\hline 1 & indudablemente_Acon034a & in & 12 & 79 & 81 & 83 & 67 & 82 & 84 \\
\hline 2 & indudablemente_Acon034a & $\mathrm{du}$ & 8 & 79 & 79 & 79 & 77 & 79 & 81 \\
\hline 3 & indudablemente_Acon034a & da & 16 & 79 & 80 & 82 & 82 & 84 & 85 \\
\hline 4 & indudablemente_Acon034a & ble & 10 & 81 & 82 & 82 & 84 & 85 & 85 \\
\hline 5 & indudablemente_Acon034a & men & 19 & 81 & 82 & 84 & 64 & 84 & 86 \\
\hline 6 & indudablemente_Acon034a & te & 5 & 83 & 83 & 83 & 65 & 81 & 85 \\
\hline
\end{tabular}

\subsection{Factores analizados}

Los factores prosódicos estudiados han sido los valores de duración y de F0 de las sílabas de los adverbios. Por su parte, esta comparación resulta algo compleja metodológicamente porque cada uno de los adverbios analizados tiene un número distinto de sílabas. Por ejemplo, el corpus incluye adverbios con cuatro sílabas ('obviamente'), con cinco ('seguramente') y con seis ('efectivamente').

Comparar los registros de duración y de F0 de todas las sílabas entre sí conduciría a errores de análisis. Por ejemplo, no pueden compararse los valores acústicos de la primera sílaba de 'obviamente' con los de las primeras sílabas de 'seguramente' o 'efectivamente'. En este caso, se estaría comparando una sílaba tónica 'ob-' con dos sílabas átonas ('se-' y 'e-' respectivamente). Esta comparación fonética no es del todo errónea, ya que, posicionalmente, establecería una comparación entre los valores de las primeras sílabas de los adverbios; sin embargo, dejaría de comparar los valores de todas las primeras sílabas tónicas de los adverbios. Debe pensarse que en 'obviamente' la primera sílaba tónica coincide con la primer sílaba del adverbio, pero que en 'seguramente' coincide con la segunda ('-gu-') y, en el caso de efectivamente, con la tercera ('-ti-').

Aun así, en estadística (Moore, 1998; Landau \& Everitt, 2004; Field, 2009), los grupos analizados deben ser homogéneos, así que, por este motivo, se han analizado los valores de la primera sílaba (sea átona o tónica), de la primera sílaba tónica, de la sílaba '-men' y de la sílaba '-te'. El único valor que se ha desdoblado es el de 'obviamente', ya que, en su caso, la primera sílaba y la primera sílaba tónica coinciden, por lo que sus registros acústicos se han duplicado en la base de datos (Tabla 3): 
Tabla 3. Estructura de los valores silábicos estudiados.

\begin{tabular}{|l|l|l|l|l|l|l|l|l|}
\hline & Dur_1 & F0_1 & Dur_ton & F0_ton & Dur_men & F0_men & Dur_te & F0_te \\
\hline obviamente & 11,18 & 86,73 & 11,18 & 86,73 & 17,55 & 85,91 & 12,00 & 90,82 \\
\hline
\end{tabular}

\section{Análisis}

\subsection{Grupos de partida}

Inicialmente, el trabajo con datos procedentes de corpus suscita una serie de problemáticas, según si los elementos que se estudian están previamente agrupados o no. En el caso de esta investigación, se ha pretendido observar la influencia de determinados factores prosódicos en la delimitación de grupos para algunos adverbios epistémicos y/o evidenciales terminados en '-mente'.

Así, los valores de F0 y de duración silábicos podrían haberse analizado de forma conjunta; por ejemplo, tras extraer las medias de F0 y de duración de la primera sílaba tónica para el adverbio 'supuestamente', se podrían haber comparado, posteriormente, con todas las medias de los diferentes adverbios analizados ('obviamente', 'seguramente'). No obstante, piénsese en los siguientes ejemplos:

(1) ${ }^{5} \mathrm{H} 1$ : porque dice/ bueno/ si por lo menos quien me va a tomar declaración se contiene la risa hasta que yo me vaya/ (RISAS)

H2: Hombre/'indudablemente'/ es una dee es una de las cosas/ a nosotros noo no es que nos- nos dé mucha risa/ precisamente/ porque se está cometiendo un- delito/ y por supuesto/ pues/ lo que pasa es quee es lógico

(2) H2: Sí

H1: Y/ después/ lleva una túnica muy bonita/ ee/ aquí lo más importante es 'indudablemente' el rostro/ la cara// es- es impresionante

En los dos ejemplos anteriores, que incluyen el adverbio 'indudablemente', se observan dos significados. En el ejemplo (1), el hablante utiliza el adverbio como un elemento de confirmación de lo dicho por el hablante anterior; es, por lo tanto, una marca de refuerzo del discurso previo. En el ejemplo (2), por su parte, el adverbio no evalúa lo dicho por el interlocutor, sino que evalúa una oración propia. De este modo, la fuente (Aikhenvald, 2011) es distinta: externa (non-visual o reported) en (1) e inferencial o propia en (2).

En general, el primer adverbio tiene una dimensión discursiva (alude a lo dicho por el interlocutor), mientras que el segundo tiene una vertiente individual, afecta generalmente a una unidad discursiva perteneciente a la intervención del propio hablante. 
Ahora bien, esa sería su diferencia semántico-pragmática. Sin embargo, si se observa su configuración prosódica, basta con leer la transcripción para estimar que el 'indudablemente' de (1) es una unidad fónica autónoma: es un grupo entonativo ubicado entre pausas. Por su parte, el 'indudablemente' de (2) es una unidad dependiente de un elemento entonativo mayor (el grupo entonativo 'aquí lo más importante es indudablemente el rostro').

Las diferencias acústicas (F0 y duración, en este caso) son evidentes: las unidades fónicas delimitadas por pausas, cuando son además relativamente breves, presentan por lo general valores prosódicos superiores a los de unidades fónicas dependientes de cláusulas entonativas cuyo ámbito es más amplio.

Un análisis estadístico no debe combinar, inicialmente, datos que procedan de grupos objetivamente distintos (Moore, 1998; Field, 2009), ya que los resultados pueden verse alterados. De tal manera y previamente al análisis estadístico, en este estudio se han dividido los datos en dos grupos: unidades fónicas completas y unidades fónicas integradas. Así, esta división ha minimizado las posibles incoherencias en los resultados obtenidos (Gráfico 2).

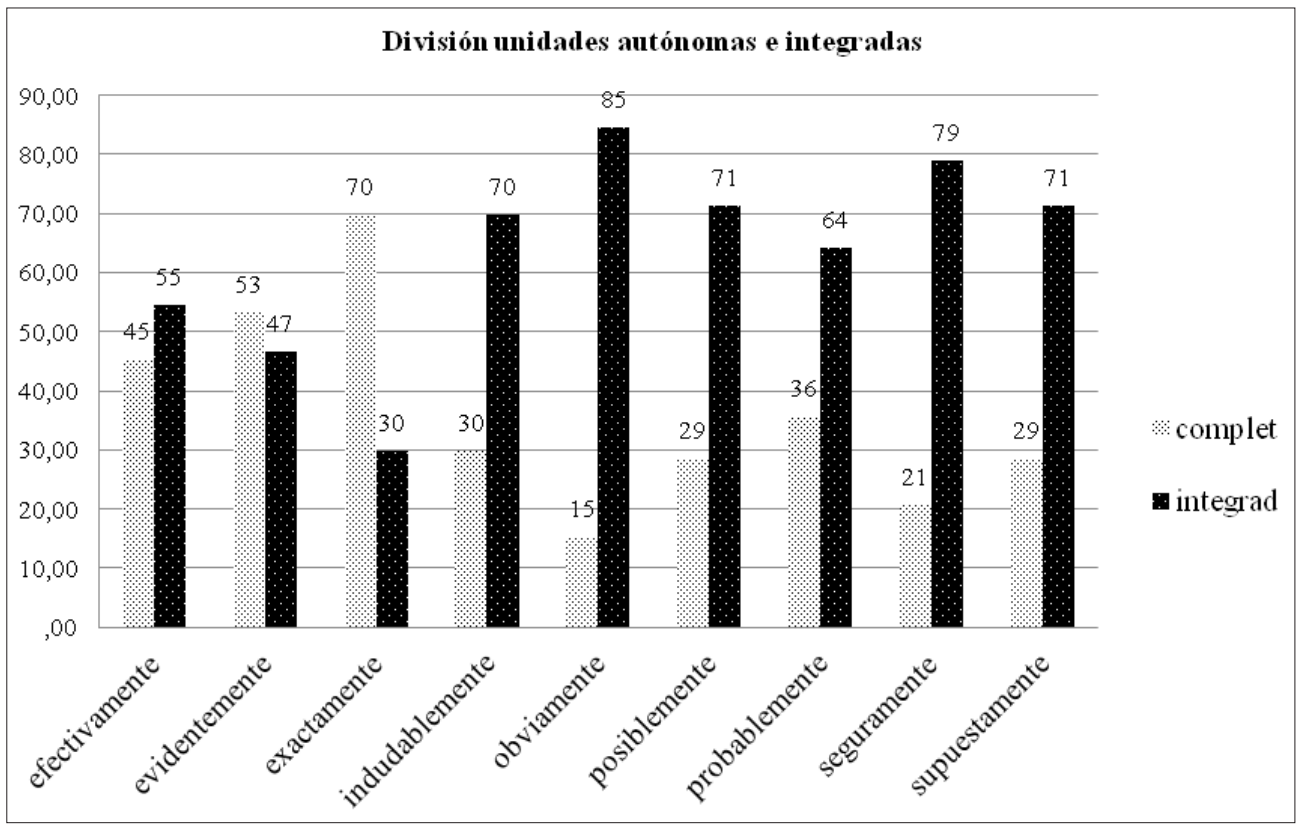

Gráfico 2. Frecuencia de unidades autónomas e integradas.

En el Gráfico 2, se observa cierta homogeneidad distribucional en los adverbios 'efectivamente' y 'evidentemente', que pueden aparecer como unidades fónicas 
autónomas y, también, como elementos fónicos integrados. Asimismo, el resto adverbios, a excepción de 'exactamente', presentan gran número de registros en posiciones no autónomas. Se trata de un hecho bastante esperable, puesto que 'exactamente', siempre que no actúe como modificador de algún adjetivo o sustantivo ('no es exactamente guapo' / 'no es exactamente profesor'), debe funcionar casi siempre como elemento de confirmación de una interlocución anterior y, por tanto, no es habitual su integración en segmentos oracionales mayores.

Así pues, en el análisis relacional de este estudio, los adverbios se han subdividido en grupos más homogéneos para asegurar una mayor confianza en los resultados estadísticos. De este modo, los adverbios se comparan siempre manteniendo la referencia sobre su inicial configuración prosódica (véanse al respecto los Gráficos $3,4$ y 5$)$.

\subsection{Análisis prosódico}

Como veíamos en el anterior apartado, los adverbios pueden aparecer en el discurso con distintas configuraciones prosódicas. Estas, normalmente, se asocian a la independencia constitutiva de la unidad en el plano fónico, es decir, con la posibilidad de aparecer o no aisladas en el discurso.

En primer lugar, se han estudiado las relaciones grupales según las variables de tiempo (duración de cada sílaba para cada adverbio), las de tono (F0 media de cada sílaba) y ambas juntas. En segundo lugar, se ha analizado el reporte gráfico (en forma de dendrograma) para observar las similitudes y diferencias entre los adverbios dentro de los grupos. Finalmente, la prueba T de Student (Landau \& Everitt, 2004; Field, 2009) ha sido utilizada para determinar las diferencias concretas entre los grupos establecidos.

\subsubsection{Variables de tiempo}

En este apartado, se observan las similtudes existentes entre los adverbios a partir de las duraciones de sus sílabas. Estas se han introducido en la base de datos como 'Dur_1' (duración de la primera sílaba), 'Dur_ton' (duración de la primera sílaba tónica), 'Dur_men' (duración de la sílaba '-men') y 'Dur_te' (duración de la sílaba '-te'). En el análisis de clúster, las cuatro variables se integran para ofrecer el informe estadístico (dendrograma del Gráfico 3). 


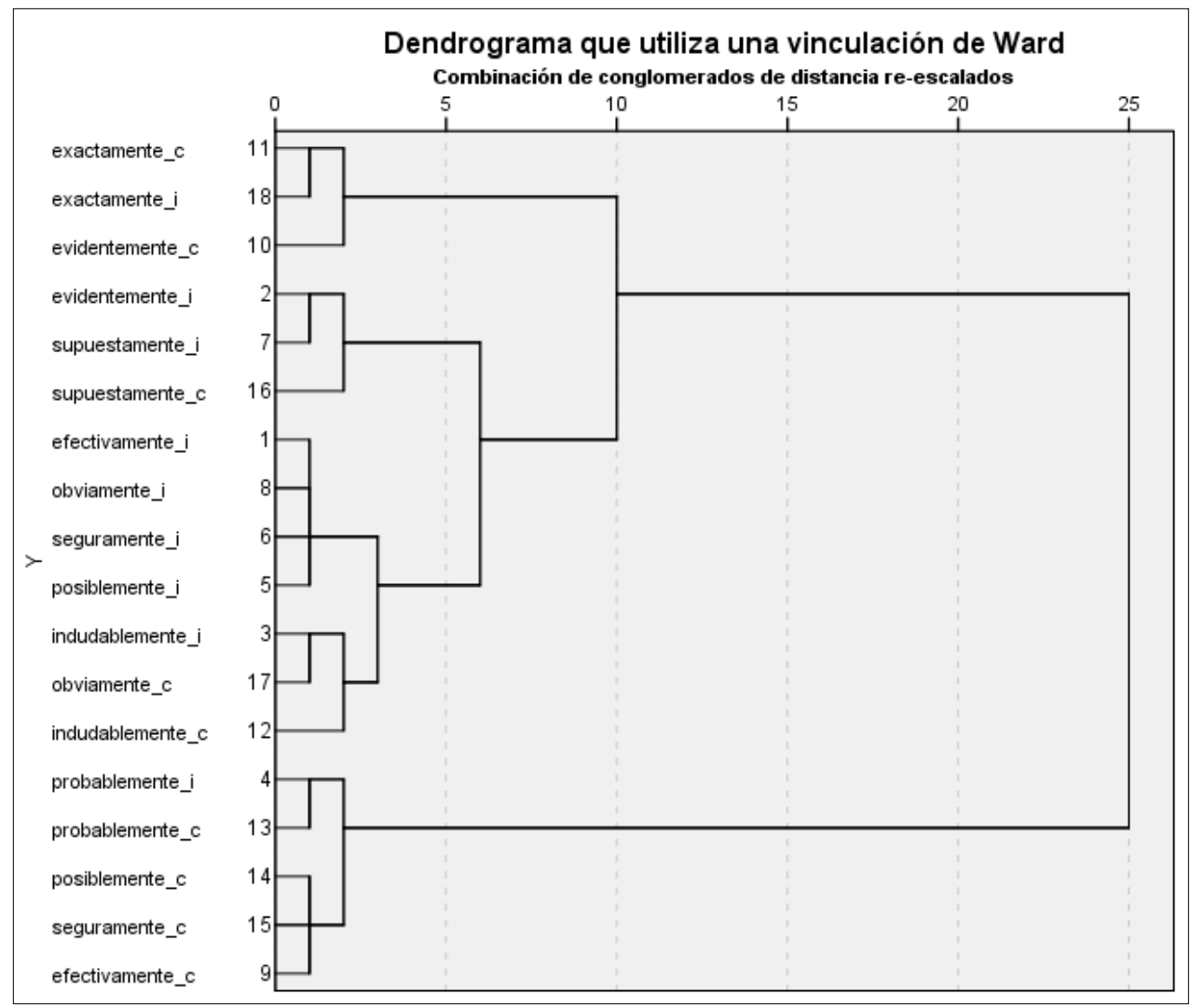

Gráfico 3. Dendrograma de los valores temporales ${ }^{6}$.

En el Gráfico 3 llama la atención que las duraciones silábicas constituyan clústeres tan variados. En general, hay dos grandes clústeres. En el primero, están la mayoría de adverbios: 'exactamente', 'supuestamente', 'posiblemente' (unidad fónica integrada), 'seguramente' (unidad fónica integrada), 'obviamente', 'indudablemente', 'evidentemente' (unidad fónica completa) y 'efectivamente' (unidad fónica integrada). En el segundo, por su parte, se sitúan 'probablemente', 'efectivamente', 'posiblemente' y 'seguramente', articulados como unidades fónicas completas.

Se ha aplicado una prueba $T$ de Student para, en el aspecto fónico, observar las similitudes y diferencias en cada una de las variables temporales analizadas. Curiosamente, los resultados determinan que las dos sílabas tónicas del adverbio (la tónica del adjetivo y la de la sílaba 'men') manifiestan una mayor duración en el primer grupo que en el segundo ( $p$ value de 0,012). Así, en el primer grupo, la sílaba tónica del adjetivo tiene una media de $13,81 \mathrm{~ms}$ y la sílaba '-men' de $17,37 \mathrm{~ms}$; por su parte, en el segundo grupo, las medias son de $8,43 \mathrm{~ms}$ y de $11,20 \mathrm{~ms}$ respectivamente. 
En el segundo clúster, tanto 'efectivamente', 'posiblemente' como 'seguramente' son unidades fónicas autónomas que, en general, refuerzan la duración silábica para transmitir confirmaciones del discurso previo (ejemplos 3 y $4^{7}$ ); normalmente, estos adverbios ocupan una posición de coda respecto al acto/subacto (Briz \& Grupo-Val. Es.Co, 2003) al que se adjuntan.

(3) MALCB2G01: que tenéis/ es que no es que funciona el chorro/ mira

MALCB2J01: es que está depurandoo// 'seguramente'

MALCB2G01: si depuraaa comooo por qué está tan sucia

MALCB2J01: el agua está limpia lo que está sucio es el fondo

(4) MALCC2G01: y va a estudiar su puta madre

MALCC2G02: y va a estudiar su puta madre/ 'efectivamente'/ y el lunes me van a coger y me van a hacer imitando sonido y estoy con los finales

(5) MAESB2J04: eso digo yo

MAESB2J01: yo lo que hago en los scouts

MAESB2J02: pero la feria de Sevilla

MAESB2J03: 'exactamente'

No obstante, otros adverbios, también autónomos fónicamente ('obviamente', 'supuestamente', 'indudablemente'), se sitúan en el primer clúster. La diferencia fónica encontrada en los grupos parece tener una explicación pragmática, ya que mientras 'obviamente', 'indudablemente', 'evidentemente' y 'exactamente' transmiten un alto grado de veracidad respecto a actos de habla cercanos y confirman de manera inmediata lo dicho por otro interlocutor (ejemplo 5), los adverbios del segundo grupo ('seguramente', 'probablemente' y 'posiblemente') expresan cautela elocutiva y, habitualmente, se adjuntan como coda a actos propios del emisor que suelen confirmar, al mismo tiempo, aseveraciones del interlocutor (ejemplos 3 y 4).

Por tanto, la duración silábica de la primera sílaba tónica y de '-men' sí resultan determinantes en el corpus analizado para discriminar los adverbios considerados puramente epistémicos por la bibliografía especializada (Kovacci, 1999; RAE, 2009, 2010; Cornillie, 2010), como 'posiblemente' o 'probablemente', frente a los considerados 'evidenciales' ('obviamente', 'supuestamente', 'indudablemente'). Estos últimos presentan duraciones más amplias en sus sílabas que los 'epistémicos'.

\subsubsection{Variables de Fo}

En cuanto a los valores de $\mathrm{F} 0$, se han incluido en el análisis de clúster las variables correspondientes a la F0 media de la primera sílaba (F0_1), de la sílaba tónica del adjetivo (F0_ton), de la sílaba '-men' (F0_men) y de la sílaba '-te' (F0_te). Las relaciones entre los adverbios se observan en el siguiente dendrograma (Gráfico 4). 


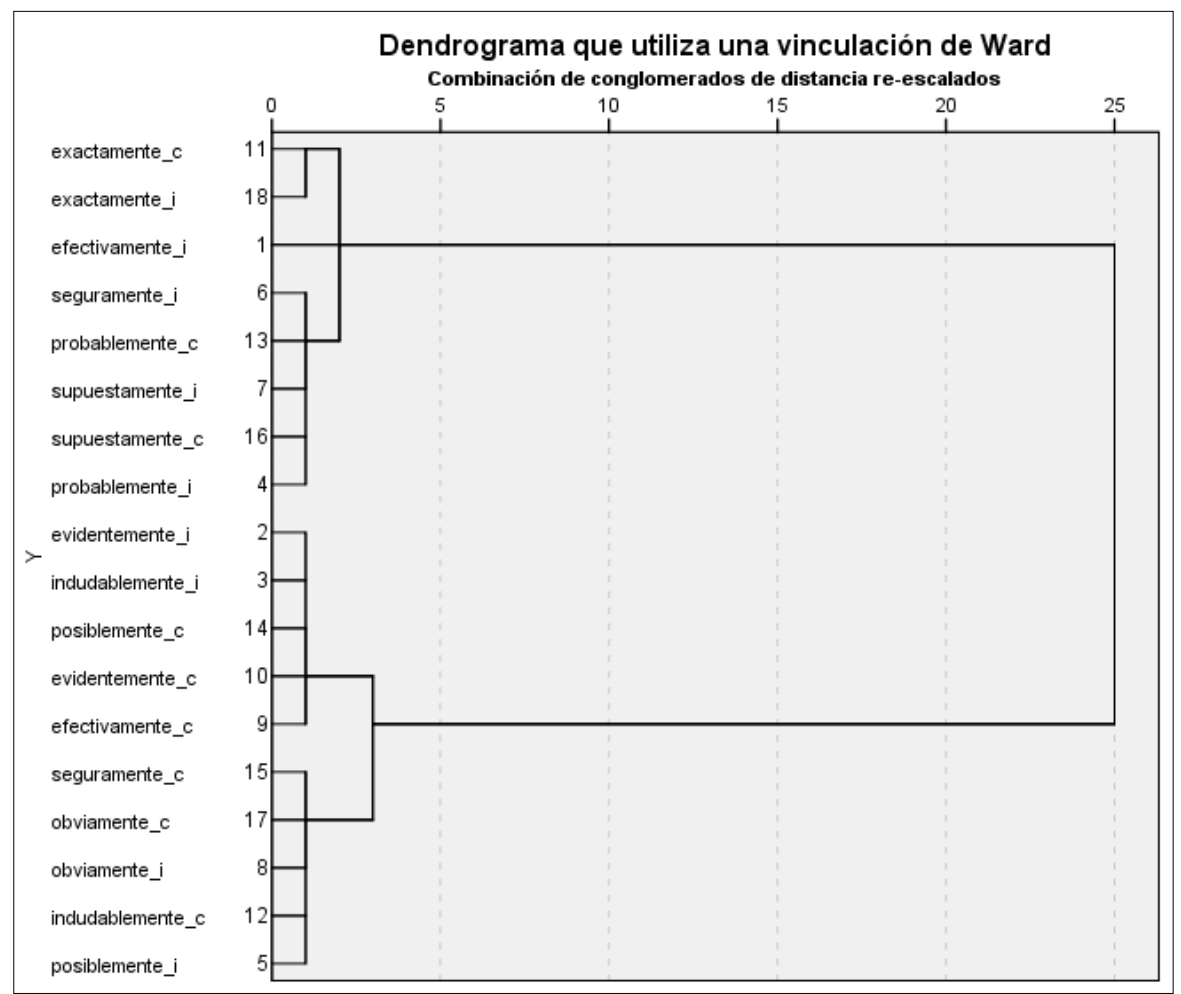

Gráfico 4. Dendrograma de los valores de Fo.

En el Gráfico 4, se observa mayor homogeneidad en los resultados obtenidos que en el apartado anterior de duración silábica. Hay dos grandes clústeres claramente establecidos. El primero lo forman 'exactamente', 'supuestamente', 'probablemente', 'efectivamente' (unidad fónica integrada) y 'seguramente' (unidad fónica integrada); el segundo está compuesto por 'evidentemente', 'indudablemente', 'posiblemente', 'efectivamente' (unidad fónica integrada), 'seguramente' (unidad fónica autónoma) y 'obviamente'.

La prueba $T$ de Student establece diferencias en los valores de F0 de todas las sílabas para los dos grupos establecidos ( $p$ value de 0,003). En concreto, el primer grupo tiene unos valores medios de 94st para la primera sílaba y la sílaba '-te', mientras que la sílaba tónica del adjetivo y la sílaba '-men' tienen medias superiores en dos semitonos (96st). Por su parte, el segundo grupo presenta registros inferiores en 10st para todas las sílabas: 83 st para la primera sílaba, 85 para la sílaba tónica del adjetivo y para la sílaba '-men' y, finalmente, 87 st para la sílaba '-te'.

En los grupos establecidos, resulta llamativo que adverbios como 'obviamente' (ejemplo 8) o 'indudablemente' tengan valores de F0 inferiores a los presentados por 
'probablemente' (ejemplo 6) o ‘supuestamente'. La intuición general parecía establecer que, a mayor grado de certeza en la valoración subjetiva de los hechos, debiera existir un mayor nivel de F0, es decir, un nivel superior de énfasis tonal. No obstante, los resultados señalan lo contrario. Asimismo, 'seguramente' actúa con valores superiores de F0 cuando es una unidad fónica integrada (ejemplo 7):

(6) P: $\left[{ }^{\circ}(\text { estará detrás })^{\circ}\right]$

C: = o estará detrás/ con el sello ese que ponen/ probablemente/[no/ es igual $\downarrow$ probablemente $=$ ] (Corpus Val.Es.Co, J.82, 757-760)

(7) $\mathrm{B}: \mathrm{la} \mathrm{Fe} / /$

D: ¿por qué no le mete una paliza?

B: porque este es GILIPOLLAS nano $\uparrow$ porque/ seguramente se ha confundido de sitio $\uparrow /$ o no s'ha acordao de qué día era nano (Corpus Val. Es.Co, 126.B ,6-10)

(8) MAESB2J03: [para saber cuál es tu grado como verte de ropa]

MAESB2J01: [estos los dos me parecen muy monos]

MAESB2J03: obviamente tenemos unos grados realmente diferentes entre chicos/ quién te parece más o menos (Corpus COLA)

En general, la variable de F0 no discrimina los grupos en la medida esperada. La intensificación pragmática que aparentemente proporcionan algunos adverbios cuando se evalúa el enunciado o el acto elocutivo ('obviamente', 'indudablemente', 'efectivamente') no se acompaña de un incremento tonal en sus sílabas; al menos, no si los comparamos con los valores de otros adverbios ('probablemente', 'supuestamente') que parecen atenuar el discurso emitido, tanto por el propio emisor como por el interlocutor.

En tales casos, el hablante utiliza la prosodia para marcar aquello que no queda directamente explícito en el nivel proposicional o, en otras palabras, en el valor semántico del elemento léxico (Cabedo \& Estellés, en prensa). Así pues, contrariamente a lo esperado, parecen elevarse más los registros de F0 en elementos que podrían considerarse atenuadores ('probablemente', 'supuestamente') que de los propiamente intensificadores ('obviamente', 'indudablemente').

Quizá en este último caso, el de adverbios que evalúan un alto grado de certeza del enunciado, el hablante no requiera de la prosodia para indicarlo, dado que el propio nivel semántico de los adverbios se convierte en un argumento suficientemente explícito. Asimismo, en adverbios que efectúan una valoración más cautelosa del discurso, el hablante hace uso de un mayor grado de énfasis tonal para asegurarse de que ese es el significado que se transmite al interlocutor. 


\subsubsection{Tiempo y Fo conjuntas}

En otros apartados, se han estudiado las variables de tiempo y las de F0 de manera separada, ya que la medida de sus valores (segundos y semitonos, respectivamente) no es la misma. No obstante, cruzar ambas variables sirve para relativizar los valores hallados con anterioridad y para observar, en general, en qué medida se combina el factor temporal con el tonal en la distribución de grupos homogéneos.

Para ello, se ha establecido otro análisis clúster (método de Ward), aunque, en esta ocasión, los valores se han estandarizado (Landau \& Everitt, 2004). Los resultados encontrados no son distintos a los estudiados en la variable de F0 (Gráfico 5).

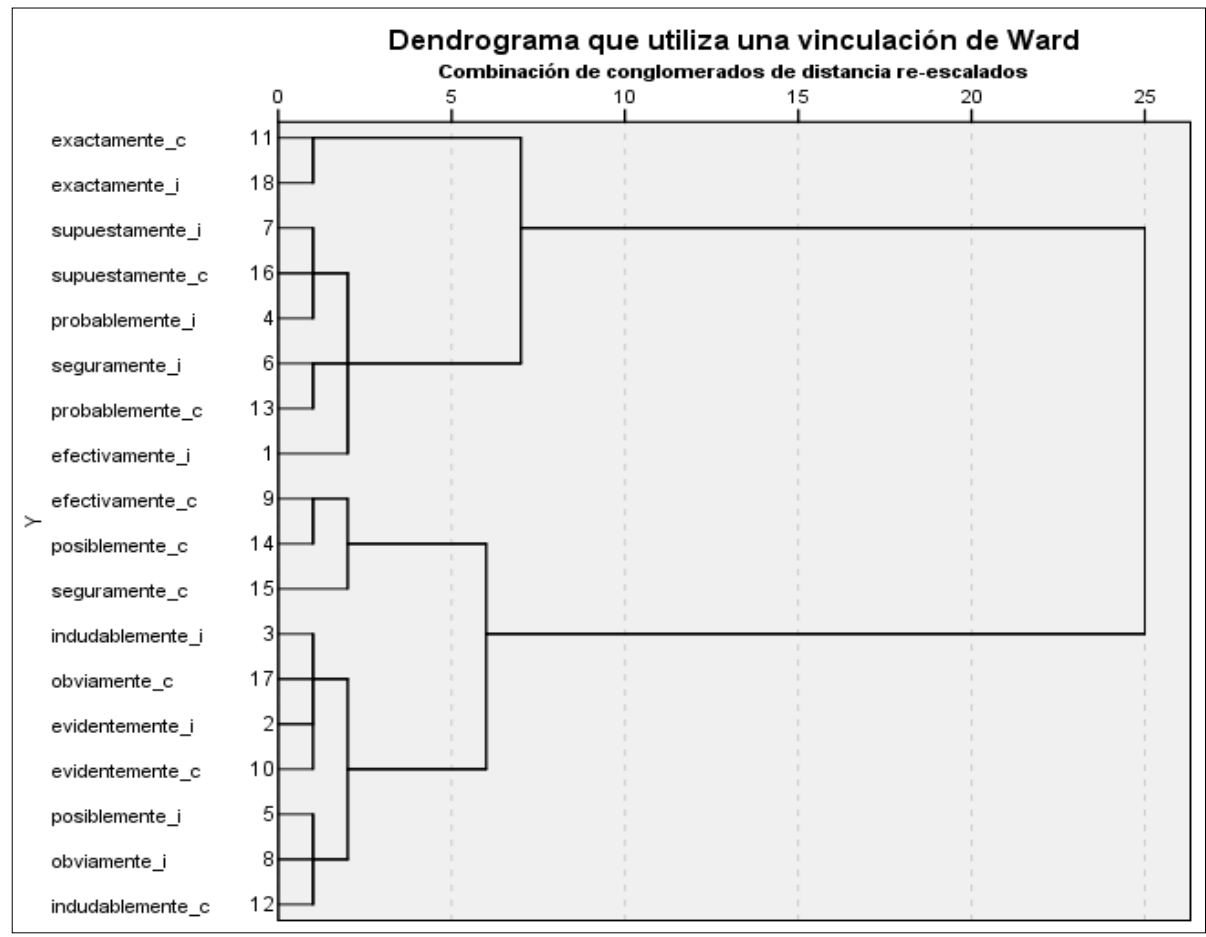

Gráfico 5. Dendrograma de los valores prosódicos conjuntos.

Mientras en el Gráfico 4 del apartado anterior los valores eran más homogéneos en la configuración de los dos clústeres generales, en el Gráfico 5 se observa mayor variación. Los grupos generales son los mismos que los establecidos para la variable de F0, es decir, hay un grupo compuesto por 'exactamente', 'supuestamente', 'probablemente', 'efectivamente' (unidad fónica integrada) y 'seguramente' (unidad fónica integrada); y, también, un segundo grupo compuesto por 'evidentemente', 'indudablemente', 'posiblemente', 'efectivamente', 'seguramente' y 'obviamente'. 
La prueba T de Student ( $p$-value de 0,014) estipula que, para los dos grupos establecidos, las variables de F0 y de duración sí presentan diferencias. Cuando se cruzan las dos variables al mismo tiempo, la variable discriminante es la de F0, pero no en el modo esperado, ya que, como sucedía en la sección 3.2.2., los adverbios que se preveían más enfáticos prosódicamente ('obviamente', 'indudablemente') siguen presentando valores más bajos que otros adverbios, como 'probablemente' o 'supuestamente', que, por su parte, se anticipaban menos marcados fónicamente.

\section{CONCLUSIONES}

Este artículo partía de distintas presuposiciones; así, se esperaba detectar, en el nivel prosódico, que adverbios como 'obviamente' o 'efectivamente' tuvieran valores de F0 y duración superiores a otros, como 'seguramente', 'probablemente' o 'posiblemente'.

Habitualmente, se presupone que el mayor grado de compromiso en la veracidad del enunciado supone una mayor conciencia por parte del hablante y que, por tanto, esa conciencia se refleja en un incremento de los valores prosódicos de F0 y de duración; por su parte, de elementos que manifiestan mayor distancia respecto a la autenticidad del discurso no se presupone un mayor control de la prosodia, sino, precisamente, valores bajos o no enfáticos. Los resultados hallados en esta investigación establecen distintas consideraciones al respecto.

La distribución grupal esperada no se ha correspondido con la determinada por el análisis de clúster. Los grupos presupuestos, según la expresión de un mayor o menor grado de compromiso en relación con la veracidad de lo dicho, eran los siguientes: \{'obviamente', 'efectivamente', 'evidentemente', 'exactamente', 'indudablemente'\} y \{'probablemente', 'posiblemente', 'seguramente', 'supuestamente'\}. En los distintos análisis efectuados en las secciones 3.2.1, 3.2.2 y 3.2.3, las variables de F0 y las de duración no han establecido dichos grupos; al menos, no con la homogeneidad esperada.

Según el análisis de la variable de duración y de F0 por separado los resultados han sido los siguientes para el corpus analizado:

1. Los adverbios 'epistémicos' ('posiblemente', 'probablemente', 'seguramente') y el adverbio 'efectivamente', como unidades fónicas completas, muestran duraciones más breves en la sílaba tónica y en la sílaba '-men'. El resto de adverbios presenta duraciones mayores.

2. Los valores tonales son mayores en adverbios que atenúan lo dicho ('probablemente', 'supuestamente'), mientras que tienden a ser menores en adverbios que intensifican la veracidad elocutiva ('obviamente', 'indudablemente'). En estos casos, el hablante parece utilizar la prosodia para 
marcar aquellos contextos en los que el valor semántico o proposicional del elemento no se considera suficientemente explícito (Cabedo \& Estellés, en prensa).

Asimismo, si se consideran los dos ámbitos estudiados (F0 y duración), los grupos generados han resultado ser los siguientes:

1. 'exactamente', 'supuestamente', 'probablemente', 'efectivamente' (unidad fónica integrada) y 'seguramente' (unidad fónica integrada);

2. 'evidentemente', 'indudablemente', 'posiblemente', 'efectivamente' (unidad fónica completa), 'seguramente' (unidad fónica completa) y 'obviamente'.

Se observan cruces entre los grupos, ya que 'exactamente' y 'efectivamente' aparecen en el primero (aparentemente compuesto por adverbios que expresan cautela elocutiva), mientras que, en el segundo grupo (adverbios que manifiestan mayor certeza en la valoración del discurso), se encuentran 'posiblemente' y también 'seguramente' (como unidad fónica autónoma).

Estas indeterminaciones, en general, parecen estar ancladas a factores de diversa índole. Por ello, futuras investigaciones deberán esclarecer el papel de los valores tonales y durativos en la discriminación de más tipos de adverbio, como los temáticos ('personalmente') o los evaluativos ('afortunadamente'), así como la influencia en el discurso de otros elementos como la posición, el registro, la frecuencia de uso e, incluso, aspectos más generales como el propio idiolecto del sujeto. 


\section{REFERENCIAS BIBLIOGRÁFICAS}

Aikhenvald, A. (2011). The grammaticalization of evidentiality. The oxford handbook of grammaticalization. Oxford: Oxford University Press.

Astruc, L. (2005). The form and function of extra-sentential elements. Cambridge Occasional Papers in Linguistics, 2, 1-25.

Astruc, L. \& Nolan, F. (2007a). Variation in the intonation of sentential adverbs in english and catalan. En C. Gussenhoven \& T. Riad (Eds.), Tones and tunes, volume I: Typological and comparative studies in word and sentence prosody (pp. 233263). Berlin: Mouton de Gruyter.

Astruc, L. \& Nolan, F. (2007b). A cross-linguistic study of extra-sentential elements. En P. Prieto, J. Mascaró \& M. J. Solé (Eds.), Segmental and prosodic issues in romance phonology (pp. 85-107). Amsterdam: John Benjamins.

Azpiazu, S. (1999). Los adverbios en -mente en español y la formación adverbial en alemán: Estudio morfológico-comparativo de esp. -mente y al. -weise. Contextos, XVII-XVIII/ 33-36, 261-277.

Briz Gómez, A. (1998). El español coloquial en la conversación: Esbozo de pragmagramática. Barcelona: Ariel.

Briz Gómez, A. (2002). Corpus de conversaciones coloquiales. Madrid: Arco-Libros.

Briz Gómez, A. \& Grupo-Val.Es.Co. (2003). Un sistema de unidades para el estudio del lenguaje coloquial. Oralia, 6, 7-61.

Cabedo, A. (2009). La segmentación prosódica en español coloquial. València: Universitat de València, Servei de Publicacions.

Cabedo, A. \& Estellés, M. (en prensa). Sobre la prosodia y su aplicación semánticopragmática en el marcador 'bueno'

Cantero Serena, F. J. (2002). Teoría y análisis de la entonación. Barcelona: Universitat de Barcelona.

Chafe, W. \& Nichols, J. (1986). Evidentiality: The linguistic coding of epistemology. Norwood, N.J.: Ablex.

Cornillie, B. (2007). Evidentiality and epistemic modality in spanish (semi-)auxiliaries : A cognitive-functional approach. Berlín/Nueva York: Mouton de Gruyter.

Cornillie, B. (2010). On conceptual semantics and discourse function. the case of spanish modal adverbs in informal conversation. Review of Cognitive Linguistics, 8(2), 300-320. 
Dendale, P. \& Tasmowski, L. (2001). Introduction: Evidentiality and related notions. Journal of Pragmatics, 33(3), 339-348.

Field, A. P. (2009). Discovering statistics using SPSS : (And sex and drugs and rock ' $n$ ' roll). Londres: SAGE.

Garcés, P. (2008). Adverbios de topicalización y marcadores de topicalización. Romanistisches Jabrbuch, 53, 355-382.

Hennemann, A. (2012). The epistemic and evidential use of spanish modal adverbs and verbs of cognitive attitude. Folia Linguistica, 46(1), 133-170.

Kaul de Marlangeon, S. (2000). El adverbio en -mente y la propiedad por él modificada como categoría evaluativa. Revista Argentina de Lingüistica, 16, 61-75.

Kotwica, D. (2011). El valor evidencial y las funciones discursivas de la particula al parecer en un corpus periodístico. Valencia: Universitat de València.

Kovacci, O. (1999). El adverbio. En I. Bosque \& V. Demonte (Coords.), Gramática descriptiva de la lengua española (pp. 705-786). Madrid: Espasa.

Landau, S. \& Everitt B. (2004). A handbook of statistical analyses using SPSS. Boca Raton, Fla./Londres: Chapman \& Hall/CRC.

López Ferrero, C. (2001). La comunicación del saber en los géneros académicos: Recursos lingüísticos de modalidad epistémico y de evidencialidad. En F. Luttokhuizen (Ed.), $V$ congrés internacional sobre llengües per a finalitats especifiques. the language of internacional communication (pp. 164-172). Barcelona: Publicacions de la Universitat de Barcelona.

Mora, A. (2011). Adverbios y prosodia. En P. Martin Butragueño (Ed.) Realismo en el análisis de corpus orales (pp. 123-147). México: El Colegio de México.

Moore, D. S. (1998). Estadística aplicada básica. Barcelona: Antoni Bosch.

Nuyts, J. (2001). Subjectivity as an evidential dimension in epistemic modal expressions. Journal of Pragmatics, 33(3), 383-400.

Pietrandea, P. (2007). The grammatical nature of some epistemic-evidential adverbs in spoken italian. Italian Journal of Linguistics, 19(1), 39-63.

Plungian, V. (2001). The place of evidentiality within the universal grammatical space. Journal of Pragmatics, 33(3), 349-357.

Quilis, A. (1993). Tratado de fonología y fonética españolas. Madrid: Gredos.

Real Academia Española. (2009). Nueva gramática de la lengua española. Madrid: Espasa. 
Real Academia Española. (2010). Nueva gramática de la lengua española. Manual. Madrid: Espasa-Calpe.

Torner Castells, S. (2005). Aspectos de la semántica de los adverbios de modo en español. Barcelona: Institut Universitari de Lingüística Aplicada. Universitat Pompeu Fabra.

Willet, T. (1988). A cross-linguistic survey of the grammaticization of evidentiality. Studies in Linguistics, 12, 57-91.

\section{NOTAS}

1 Algunos planteamientos teóricos señalan que esa fuente puede ser de distinto tipo: visual, auditiva, inferencial... (Aikhenvald, 2011).

2 El corpus Valesco tiene una edición en papel (Briz, 2002) y una edición online (http://www. fonocortesia.es/corpusval). El corpus COLA puede encontrarse en el siguiente enlace: http:// www.colam.org/om_prosj-espannol.html

3 Los textgrids son archivos de texto que permiten alinear visualmente la transcripción del fragmento con el audio.

4 Disponible en: http://uk.groups.yahoo.com/group/praat-users/files/Daniel_Hirst/analyse_tier.praat

5 Los dos ejemplos se han extraído del Corpus oral de referencia del español contemporáneo de Marcos Marín y han sido adaptados a las convenciones del sistema de transcripción del Grupo Val.Es.Co (Briz, 2002).

6 Para mejorar la comprensión y facilitar la lectura, las unidades autónomas llevan el sufijo _c, que significa 'completo'; por su parte, las unidades no autónomas presentan el sufijo _ $i$, que significa 'integrado'.

7 Extraídos del Corpus COLA y adaptados al sistema de transcripción del grupo Val.Es.Co (Briz, 2002). 